\title{
Survivors: Wie Schmerz den Alltag beeinträchtigt
}

Überlebende einer Krebserkrankung im Kindesalter haben als Erwachsene ein erhöhtes Risiko für Schmerzen und damit verbundene funktionelle Beeinträchtigungen. Eine US-Studie lässt erkennen, wie stark der Schmerz den Alltag der Betroffenen beeinflusst [1].

Krebserkrankungen im Kindesalter können zunehmend erfolgreich behandelt werden. Heute leben mindestens fünf Jahre nach der Diagnose noch etwa $84 \%$ der betroffenen Kinder und Jugendlichen. Doch selbst wenn die Patienten offiziell als geheilt gelten, entwickeln rund $95 \%$ von ihnen im Lauf der Zeit körperliche, neurokognitive oder psychosoziale Beeinträchtigungen. Viele klagen im Erwachsenenalter über Schmerzen, nicht selten werden die Beschwerden chronisch. Wie viele ehemalige Krebspatienten unter Schmerzen leiden und inwieweit diese verschiedene Aspekte des Alltags beeinflussen, haben jetzt Ingrid Tonning Olsson vom St. Jude Children's Research Hospital, Memphis, und Kollegen untersucht.

Primäre und sekundäre Endpunkte In die Studie eingeschlossen wurden 2836 Krebsüberlebende ab 18 Jahren, deren Diagnose mindestens zehn Jahre zurücklag (im Schnitt 23,7 Jahre nach der Diagnose). Die Studienteilnehmer waren durchschnittlich 32,2 Jahre alt. Die Schmerzstärke wurde auf einer 6-Punkte-Likert-Skala auf der Basis des SF-36 erfasst. Sekundäre Studienendpunkte waren Neurokognition (Tests zu Aufmerksamkeit, Erinnerungsvermögen, exekutiver Funktion, Verarbeitungsgeschwindigkeit), körperliche Funktion (Tests zu aerober Ausdauer, Flexibilität, Beweglichkeit, Kraft, Gleichgewicht, adaptiven Körperfunktionen), sozioökonomische Faktoren sowie die gesundheitsbezogene Lebensqualität (HRQoL).

Etwa $36 \%$ der Studienteilnehmer waren im Kindesalter an einer Leukämie erkrankt gewesen, $9 \%$ hatten einen ZNS-Tumor überlebt, $21 \%$ ein Lymphom, 7 \% ein Ewing-Sarkom oder
Osteosarkom und $25 \%$ waren Überlebende anderer solider Tumoren. $62 \%$ der pädiatrischen Krebspatienten waren operiert worden, $85 \%$ hatten eine Chemotherapie erhalten, $32 \%$ eine kraniale und $25 \%$ eine nichtkraniale Strahlentherapie.

\section{$18 \%$ im Alltag vom Schmerz beeinflusst}

$27 \%$ der ehemaligen Krebspatienten berichteten über moderate bis sehr starke körperliche Schmerzen, $24 \%$ über Migräne oder starke Kopfschmerzen (vs. $15 \%$ bzw. $19 \%$ in der Kontrollgruppe mit 343 vergleichbaren Personen ohne kindliche Krebserkrankung). $65 \%$ derjenigen, die Schmerzmittel einnahmen, erreichten keine Analgesie. Schmerzen, die ihr tägliches Leben moderat bis extrem beeinträchtigten, hatten $18 \%$ der Krebsüberlebenden gegenüber $8 \%$ der Kontrollgruppe; damit waren sie gemäß der adjustierten Analyse 3,4mal so häufig betroffen. Kein signifikanter Unterschied ergab sich bei der Häufigkeit von Schmerzen, die keinen Einfluss auf den Alltag hatten.

Am häufigsten traten Schmerzen bei Überlebenden von Weichteilsarkomen, Non-Hodgkin-Lymphomen, Ewing-Sarkomen, Osteosarkomen und nach Amputationen und/oder Extremitäten-schonenden Operationen auf. Auch bestehende chronische Krankheiten oder Übergewicht erhöhten das Risiko für beeinträchtigenden Schmerz. Zudem waren überwiegend Personen über 30 Jahre betroffen.

Bei Krebsüberlebenden mit täglichen Schmerzen ergaben sich gegenüber schmerzfreien Studienteilnehmern ein erhöhtes Risiko für Beeinträchtigungen in folgenden Bereichen: Aufmerksam- keit (Risikoerhöhung 65-99\%), Erinnerungsvermögen (65\%), exekutive Funktion (73\%) und Verarbeitungsgeschwindigkeit $(70 \%)$. Zudem beeinflusste der Schmerz nahezu alle Aspekte der körperlichen Funktionsfähigkeit. Am stärksten betroffen war die aerobe Ausdauer. Auch die Arbeitsfähigkeit in Beruf und Schule war massiv beeinträchtigt. Fast sechsmal so häufig wie bei schmerzfrei Überlebenden war eine Unterstützung im Alltag erforderlich. Gleichzeitig verschlechterte sich die körperliche und psychische HRQoL, z. B. war das Risiko für eine reduzierte psychische Gesundheit verdoppelt, das Risiko für Schwierigkeiten beim Vollenden vom Aktivitäten aufgrund von körperlichen Funktionseinschränkungen sogar versechsfacht.

\section{Regelmäßiges Schmerz-Screening} Auch wenn hierzu bislang keine randomisierten Studien existierten, so Tonning Olsson und Kollegen, könnte eine kognitive Verhaltenstherapie den Patienten möglicherweise helfen. Deshalb lohne sich ein regelmäßiges Schmerz-Screening.

\section{Literatur}

1. T Olsson I et al (2020) Pain and Functional Outcomes in Adult Survivors of Childhood Cancer: A Report From the St. Jude Lifetime Cohort Study. Cancer

Hinweis des Verlags. Der Verlag bleibt in Hinblick auf geografische Zuordnungen und Gebietsbezeichnungen in veröffentlichten Karten und Institutsadressen neutral.

Paediatr. Paedolog. 2021 · 56:82 https://doi.org/10.1007/s00608-02100877-7

(c) Springer-Verlag GmbH Austria, ein Teil von Springer Nature 2021 